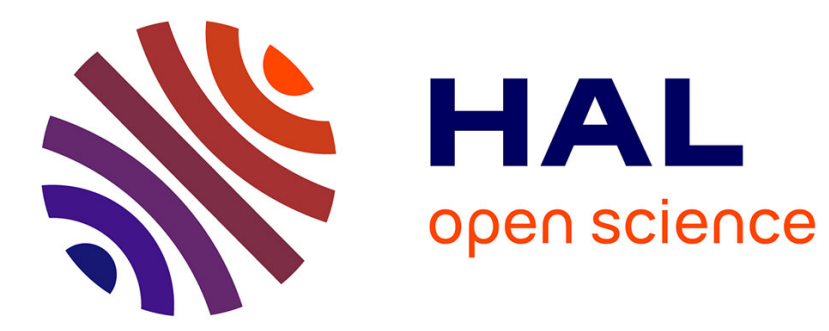

\title{
Les armes du Hezbollah: terrorisme, droit à la résistance et principe de légalité
}

\author{
Nicolas Dot-Pouillard
}

\section{To cite this version:}

Nicolas Dot-Pouillard. Les armes du Hezbollah : terrorisme, droit à la résistance et principe de légalité. Confluences Méditerranée, 2017, Terrorisme(s), 3 (102), pp.89-102. 10.3917/come.102.0089 . halshs01619355

\section{HAL Id: halshs-01619355 \\ https://shs.hal.science/halshs-01619355}

Submitted on 19 Oct 2017

HAL is a multi-disciplinary open access archive for the deposit and dissemination of scientific research documents, whether they are published or not. The documents may come from teaching and research institutions in France or abroad, or from public or private research centers.
L'archive ouverte pluridisciplinaire HAL, est destinée au dépôt et à la diffusion de documents scientifiques de niveau recherche, publiés ou non, émanant des établissements d'enseignement et de recherche français ou étrangers, des laboratoires publics ou privés. 
Les armes du Hezbollah : terrorisme, droit à la résistance et principe de légalité ${ }^{1}$

Nicolas Dot-Pouillard est Chercheur associé à l'Institut français du Proche-Orient (Ifpo) et Core-Researcher au sein du programme Wafaw - European Research Council. II est l'auteur de La Mosaïque éclatée. Une histoire du mouvement national palestinien (19932016), Editions Acte sud, Arles et Institut des études palestiniennes, Beyrouth, 2016.

Résumé :

Terroriste, ou libérateur? Depuis le milieu des années 1980, le Hezbollah suscite des interprétations divergentes. Pour les uns, c'est un mouvement terroriste, qui doit être éradiqué : tel est le point de vue d'Israël ou des Etats-Unis, rejoints, depuis 2016, par l'Arabie saoudite. Pour les autres, le Hezbollah est condamnable : mais il y a un principe de réalité. L'Union européenne distingue ainsi entre une branche politique et une branche militaire du Hezbollah. Il est possible de dialoguer avec la première, il faut en finir avec la seconde. Enfin, il y a la narration du Hezbollah et de ses partisans : le Hezbollah n'est pas un mouvement terroriste, puisque ses armes bénéficient d'un principe de légalité au Liban. Son opposition à l'Etat islamique lui permet enfin d'intégrer la rhétorique de l'anti-terrorisme à son arsenal conceptuel. Les divergences d'interprétations au sujet du Hezbollah montrent surtout que l'adjectif terrorisme relève moins de l'ordre du droit, que de l'ordre du dire.

\footnotetext{
${ }^{1}$ Cet article est le fruit de recherches menées dans le cadre du programme Wafaw-ERC. Les opinions et
} analyses de cet article sont celles de son auteur, non de l'institution qui l'a financé. 
Dans la galaxie islamiste, le Hezbollah libanais suscite des interprétations divergentes. Il y aurait, pour les uns, le Hezbollah de mai 2000 et de la guerre de juillet et août 2006 : celui qui a libéré le sud-Liban de l'occupation israélienne (1978-2000), puis affaibli Tsahal lors de l'été 2006, en un combat asymétrique parfois comparé à un "Diên Biên Phu arabe ${ }^{2}$ ». Le Hezbollah serait l'héritier: de la geste anti-impérialiste d'un Jamal Abdel Nasser, et du mouvement de libération nationale palestinien. Fils de la révolution iranienne de 1979, il a joué le jeu du compromis confessionnel libanais, si cher à l'esprit du Pacte national de 1943 : par exemple en contractant une alliance avec le Courant patriotique libre (CPL), la principale formation chrétienne, conduite par le futur président Michel Aoun, en février 2006, ou en laissant les manettes du gouvernement à son adversaire Saad Hariri, en décembre 2016.

L'adjectif « chiite » ne suffirait pas à le définir : ne serait-il pas l'un des constants soutiens de partis politiques palestiniens, donc sunnites ${ }^{3}$ ? Il serait enfin l'envers même du terrorisme : son intervention militaire en Syrie, depuis 2012, se ferait dans le cadre d'une guerre défensive. Protégeant les frontières orientales du Liban, il serait l'un des éléments moteurs d'une nouvelle équation anti-terroriste libanaise, l'associant notamment à l'armée.

Cette vision d'un Hezbollah "résistant » et «nationaliste », imposant un rapport de force militaire régional face à Israël, défiant l'Arabie saoudite, garant d'une souveraineté libanaise menacée par deux ennemis exclusifs - l'armée israélienne et les groupes salafistesdjihadistes- a ses partisans au Liban: pas seulement chez des chiites soudés par une communauté de destin avec le Parti de Dieu depuis le milieu des années 1980, mais aussi dans une partie de la rue chrétienne. Dans le monde arabe, le soutien du Hezbollah au régime syrien lui a sans doute fait perdre l'amitié que pouvait lui témoigner par le passé les organisations proches des Frères musulmans - à l'exception peut-être du Hamas palestinien. Il a pourtant gardé des appuis ailleurs : à gauche, dans ce qu'il reste du nationalisme arabe, ou dans une partie des opinions publiques pour qui le paradigme de l'opposition au «danger salafiste » tient lieu de principale grille de lecture politique. L'Union générale tunisienne du travail (UGTT), auréolée de son prix Nobel de la paix en décembre 2015, a fermement condamné, en mars 2016, la décision du Conseil des ministres arabes de l'intérieur, réunis à Tunis, de classer le Hezbollah sur sa liste des organisations terroristes.

\section{Terrorisme : l'ordre du droit, l'ordre du dire}

A la narration «résistante» des partisans d'Hassan Nasrallah, le secrétaire-général du Hezbollah, s'opposerait celle de ses contempteurs. L'adjectif «terroriste » est rarement utilisé au Liban par les adversaires du Hezbollah, de peur de remettre en cause le précaire alliage institutionnel qui associe, au nom même du Pacte national, les ennemis d'hier et d'aujourd'hui. Mais, depuis plus d'une dizaine d'années, des membres de la Coalition du 14 mars - du Courant du futur, sunnite, aux Forces libanaises, maronites, en passant par le Parti socialiste progressiste druze (PSP) de Walid Joumblatt- ont accusé le Hezbollah d'être directement responsable de l'assassinat de l'ancien Premier ministre Rafiq Hariri, en février 2005. Leurs hostilité au Hezbollah s'appuie sur le droit : en l'occurrence, celui du Conseil de sécurité des Nations Unies, qui, le 30 mai 2007, «crée d'autorité le Tribunal spécial pour le Liban par la résolution 1757 », "pour juger les responsables de l'assassinat de Rafiq Hariri (le 14 février 2005) et d'une vingtaine d'autres personnes ${ }^{4} \gg$. En août 2013, c'est le même

2Adlène Meddi et Walid Charara, « La bataille du Liban. Un Diên Biên Phu arabe », Al Watan, 7 août 2006.

${ }^{3}$ Nicolas Dot-Pouillard et Wissam Alhaj, "Pourquoi le Hamas et le Hezbollah restent quand même alliés? Au-delà de la crise syrienne et du clivage entre sunnites et chiites», Orient XXI, 9 mars 2015, http://orientxxi.info/magazine/pourquoi-le-hamas-et-le-hezbollah-restent-quand-meme-allies, 0831

${ }^{4}$ Maria Luisa Cesoni et Damien Scalia, «Juridictions pénales internationales et Conseil de sécurité : une justice politisée», Revue québécoise de droit international, 25.2, 2012, pp 66-67, en ligne, https://www.sqdi.org/wp-content/uploads/RQDI_25-2_2_Cesoni-Scalia.pdf 
Tribunal spécial pour le Liban qui émet des mandats d'arrêts internationaux contre quatre membres du Hezbollah dans le cadre de l'enquête sur l'assassinat de Rafiq Hariri, et qui ouvre un procès officiel à leur encontre en janvier 2014.

Le mot terroriste est utilisé plus explicitement par les Israéliens : pour eux, le Hezbollah l'est au même titre que l'ensemble des formations politiques palestiniennes. Le mouvement chiite est également placé sur la liste des organisations terroristes de plusieurs états, comme les Etats-Unis, le Canada, ou l'Australie. En juillet 2013, 28 ministres des Affaires étrangères décident de classer la branche militaire du Hezbollah sur la liste des organisations terroristes de l'Union européenne. La guerre civile en Syrie, tout comme l'entrée en guerre de l'Arabie saoudite au Yémen depuis mars 2015, les tensions récurrentes entre les pays membres du Conseil de coopération du Golfe (CCG) et l'Iran, permettent de franchir un cap : en mars 2016, le CCG et le Conseil des ministres arabes de l'Intérieur qualifient également le Hezbollah d'organisation terroriste. Les reproches à l'encontre du Parti de Dieu dessinent l'image d'un parti qui serait la branche politique d'une armée, d'un mouvement plus attaché au devenir des chiites de la région qu'à la souveraineté libanaise, d'un Etat dans l'Etat, soucieux d'abord de ses armes - comme l'aurait révélé la «prise» de Beyrouth en mai 2008 contre les partisans du Courant du futur de Saad Hariri ${ }^{5}$ - et d'un intermédiaire iranien aux finances opaques.

Le terrorisme relève sans doute moins de l'ordre du droit que de l'ordre du dire : dans le cas du Hezbollah, l'adjectif terroriste est l'objet d'une guerre des narrations. Elles sont au nombre de trois. La première est éradicatrice : partagée par les Etats-Unis, Israël et l'Arabie saoudite, elles fait du Hezbollah l'incarnation de l'ennemi absolu. C'est l'équivalent chiite du djihadisme de l'Etat islamique (IS). La seconde narration, celle de l'Union européenne ou de la France, est hésitante : terroriste, le parti de Dieu le serait incontestablement, mais il serait possible de distinguer sa branche politique de sa branche militaire. La troisième narration est celle du Hezbollah et de ses soutiens: cette narration «résistante » ne fait pas que réfuter le terme de terroriste. Elle se prévaut de l'ordre d'un principe de légitimité et de légalité- le «droit à la résistance » reconnu par les différentes déclarations d'investiture gouvernementale depuis 1990- tout en intégrant progressivement, depuis 2011, le vocabulaire même de l'anti-terrorisme à son arsenal conceptuel.

\section{L'ennemi à abattre}

Alain Joxe comparait l'administration républicaine de Georges W. Bush à une «agence de notation doctrinaire », définissant «un ennemi stratégique unifié ${ }^{6} »$. Dans l'échelle de notation américaine du «terrorisme global», ce ne sont plus l'Irak de Saddam Hussein ou les Talibans afghans qui font figure de principaux ennemis à abattre. Le discours de Donald Trump tenu à Ryad, le 21 mai 2017, lors d'un Sommet islamique arabo-américain, a désigné les adversaires principaux des Etats-Unis dans la région : l'Iran, le Hezbollah, le Hamas palestinien, Al-Qaeda et l'Etat islamique. Téhéran est accusé de tous les maux : d'avoir «déstabilisé la Syrie », "de financer, armer et entraîner des terroristes, des milices et des groupes extrémistes, du Liban à l'Irak, en passant par le Yémen ${ }^{7}{ }$.

Donald Trump a énoncé un classique de la politique américaine dans la région depuis les années 1980, à savoir la dénonciation de l'Iran et de son principal allié au Liban. Certes, l'administration Obama s'est faite plus conciliante avec Téhéran, en obtenant, en juillet 2015, un compromis sur le nucléaire iranien, s'attirant les foudres israéliennes et saoudiennes. Mais

\footnotetext{
${ }^{5} \mathrm{~A}$ la suite d'une décision du premier Ministre Fouad Siniora de démanteler le réseau de communication interne du Hezbollah, et de limoger le responsable de la sécurité de l'aéroport de Beyrouth, proche du parti, de violents combats éclatent au Liban du 7 au 14 mai 2008, opposant le Hezbollah au Courant du Futur.

${ }^{6}$ Alain Joxe, Les guerres de l'empire global. Spéculations financières, guerres robotiques, résistances démocratiques, Editions La Découverte, Paris, 2012, p 46.

7 "Transcript of Trump's Speech in Saudi Arabia”, CNN Politics, May 21, 2017, http://edition.cnn.com/2017/05/21/politics/trump-saudi-speech-transcript/index.html
} 
les pressions américaines sur le Hezbollah se sont bien accrues sous l'ère Obama. En décembre 2015, l'adoption d'un Hezbollah International Financing Prevention Act (HIFPA) par le Congrès américain, voté par les Démocrates et les Républicains, a obligé la Banque centrale libanaise à clôturer des comptes liés au Hezbollah. Le rapport annuel 2016 du Bureau contre-terroriste du Département d'Etat américain fait du Hezbollah, «avec une aide considérable de l'Iran, l'organisation terroriste la plus efficace au Liban ${ }^{8} »$.

L'insistance de Donald Trump sur le Hezbollah et l'Iran constitue tout de même un tournant : avec l'Arabie saoudite et Israël, la convergence stratégique est désormais totale. C'est la grande différence avec l'ère Obama, dont les relations avec Ryad et Tel Aviv s'étaient peu à peu détériorées, suite à l'accord sur le nucléaire iranien.

\section{Le pari saoudien}

Les Saoudiens ont en effet franchi un cap : la guerre de moins en moins froide avec l'Iran, leur enlisement militaire au Yémen, leur soutien à certaines factions de l'opposition armée syrienne, les ont amené à faire du Hezbollah une cible évidente. Ils sont à l'offensive diplomatique contre la formation chiite libanaise depuis mars 2016. Ils ont obtenu que le Conseil de coopération du Golfe (CCG) classe le Hezbollah comme organisation terroriste. Ensuite, ils ont fait voter la même décision par le Conseil des ministres arabes de l'Intérieur, réunis à Tunis. C'est une guerre globale qu'ils mènent : elle est également médiatique. En décembre 2015, le réseau satellitaire Arabsat, basé à Ryad, suspend la diffusion de la chaîne télévisée du Hezbollah, Al-Manar.

L'offensive saoudienne à l'encontre du Hezbollah est inédite. Certes, dans la seconde moitié des années 2000, les tensions entre Ryad et le Hezbollah étaient récurrentes : mais elles demeuraient contenues. En poussant le CCG et la Ligue arabe à durcir leur position contre l'organisation chiite, le roi Salman a parié sur une perte de crédit du Hezbollah à la suite de son intervention militaire en Syrie, et compté sur une Ligue arabe dont le régime syrien a été expulsé en 2011 - ses soutiens étatiques sont maigres, seule l'Algérie soutenant officiellement Damas. Mais c'est un pari hasardeux. L'Arabie saoudite a mis en porte à faux son principal allié au Liban, le Courant du futur de Saad Hariri, dont le retour aux commandes du gouvernement libanais était encore conditionné, en mars 2016, par une alliance avec les composantes chrétiennes de la Coalition du 8 mars - donc avec les propres partenaires du Hezbollah. L'Egypte a certes cédé aux demandes saoudiennes : mais elle joue un double jeux, entretenant des liens discrets avec le Hezbollah au nom de la commune lutte contre le terrorisme : en février 2016, Hassan Ezzedine, chargé des affaires arabes du Hezbollah, s'est rendu au Caire pour présenter ses condoléances à la famille de Muhammad Hassanein Heikal, un intellectuel égyptien proche des autorités. Quant au CCG, il est divisé : pour preuve la crise ouverte entre Doha et Ryad en juin 2017, dont les Iraniens espèrent tirer profit à l'avenir.

\section{L’honnête courtier des Israéliens}

Un Etat pense cependant avoir marqué des points contre le Hezbollah : c'est Israël. Le Hezbollah et l'Iran sont ses ennemis principaux, loin devant l'Etat islamique. Dans un article paru le 21 mai 2017 sur le site de la très conservatrice chaine américaine Fox News, l'ambassadeur israélien à l'ONU, Danny Danon, définit sans ambiguïté l'ordre des priorités israéliennes - saluant la nouvelle doctrine stratégique de Donald Trump : l'Iran d'abord, le Hezbollah ensuite, le Hamas enfin. Le cas de l'Etat islamique semble anecdotique - il n'est

8 "Country report on Terrorism 2016", United States State Department Publication, Bureau of Counterterrorism, July 2017, https://www.state.gov/documents/organization/272488.pdf 
cité qu'une fois par l'ambassadeur israélien ${ }^{9}$, et l'inquiète moins que les tentatives de pénétrations iraniennes en Syrie et au Yémen. L'Iran est un ennemi proche et lointain : il agirait par «proxies » (intermédiaires), au nord comme au sud d'Israël, par Hezbollah et Hamas interposés.

Dans les années 2000, le terme «terroriste » accolé au Hezbollah demeurait encore tabou dans le monde arabe, au grand dam d'Israël : les pays membres de la Ligue arabe craignaient alors de s'aliéner des opinions publiques impressionnées par les succès militaires du Hezbollah contre l'armée israélienne. Les guerres civiles syriennes et yéménites ont depuis modifié la donne : en devenant des "conflits internes internationalisés ${ }^{10}$ » et régionalisées, elles ont ouvert de nouvelles perspectives stratégiques pour Tel Aviv. En saluant le discours de Donald Trump tenu à Ryad, Israël peut maintenant compter, dans sa guerre de trente ans contre le Hezbollah, sur un honnête courtier saoudien qui plaide désormais auprès des Américains et de la Ligue arabe sa cause principale : l'éradication du Hezbollah, menace stratégique majeure sur ses frontières nord.

\section{Le politique et le militaire}

Le 26 février 2000, Lionel Jospin, alors premier Ministre socialiste, fait face aux étudiants palestiniens de l'Université de Birzeit, en Cisjordanie, venus manifester contre sa présence sur le campus. Ils lui reprochent notamment d'avoir qualifieé le Hezbollah d'organisation «terroriste »- le sud-Liban est alors occupé par Israël. Treize ans plus tard, c'est un autre membre du Parti socialiste - Laurent Fabius, alors ministre des Affaires étrangères- qui soutient la proposition britannique de classer le Hezbollah sur la liste européenne des organisations terroristes. En juin 2013, un tournant est acté : au même titre que le Hamas, le Hezbollah est désormais considéré par l'Union européenne comme terroriste.

\section{Des relations dégradées}

Laurent Fabius ne s'en cache pas : la présence militaire du Hezbollah en Syrie a fortement pesé dans le consensus des 28. Le Royaume-Uni et les Pays-Bas réclamaient des sanctions contre le Hezbollah depuis des années. C'est également la Bulgarie, membre de l'UE depuis 2007, qui a favorisé cette décision: elle accuse notamment le Hezbollah d'être responsable d'un attentat commis à Burgas contre des civils israéliens, le 18 juillet 2012 - ce dont se défend depuis le parti libanais. Toujours est-il que l'année 2013 a été une rupture: les Américains et les Israéliens ont longtemps reproché aux Européens leurs tiédeurs face au Hezbollah. En février 2000, les propos de Lionel Jospin n'étaient pas ceux de Jacques Chirac $^{11}$. Plus d'une décennie plus tard, l'adjectif terroriste fait consensus en France et en Europe.

Cette décision est l'aboutissement logique de relations dégradées entre le Hezbollah et les puissances européennes depuis plus de 12 ans. L'Union européenne demandait un retrait militaire syrien - acté en 2005- alors que le Hezbollah s'y opposait. La résolution onusienne 1559 de septembre 2004 était à l'initiative de la France et des Etats-Unis. Elle demandait le désarmement du Parti de Dieu : il ne s'y est naturellement jamais plié. Depuis 2007, l'Union européenne a soutenu les travaux du Tribunal spécial pour le Liban (TSL), installé à La Haye: le Hezbollah est dans son collimateur principal. Les principales puissances européennes ont condamné la « prise » de Beyrouth par l'organisation chiite, en mai 2008, après des combats l'opposant aux miliciens du Courant du futur. L'intervention militaire

\footnotetext{
9 Dany Danon, "Iran Remains the Crucial Issue", Fox News Opinions, May 21, 2017, http://www.foxnews.com/opinion/2017/05/21/israeli-ambassador-on-trump-in-middle-east-iranremains-crucial-issue.html

${ }^{10}$ Charles Tilly et Sydney Tarrow, Politique(s) du conflit. De la grève à la révolution, Les Presses de Sciences Po, Paris, 2008, p 256.

${ }^{11}$ Alain Gresh et Hélène Aldeguer, Un chant d'amour. Israël-Palestine, une histoire française, Editions La Découverte, Paris, 2017, p 153.
} 
progressive du Hezbollah en Syrie depuis 2012 pour soutenir Bashar al-Assad fut un point d'orgue : l'Union européenne s'étant rangé du côté des opposants, la logique des axes fit le reste. Il n'est alors pas étonnant que dans cette classification du Hezbollah comme organisation «terroriste », deux Etats - et non des moindres- y soient encore tout à fait hostiles : la Russie, et la Chine. La classification d'un mouvement comme terroriste n'est pas affaire de valeurs, et il n'y a pas d'ennemis réellement universels.

\section{Une affaire de nuances}

La position européenne - et donc française- se distingue cependant de celle adoptée par les Etats-Unis, Israël, le Canada ou l'Arabie saoudite. C'est la branche militaire du Hezbollah que l'UE condamne - et non sa branche politique. La nuance est de taille, et la logique est moins éradicatrice à Bruxelles qu'à Washington et Tel Aviv. Le Hezbollah est alors un Janus au deux visages : il serait possible de dialoguer avec les politiques, et de condamner les militaires. Dans cette affaire, sans doute nul n'est tout à fait dupe : organiquement, le Hezbollah lui-même se défend d'une telle dichotomie. En réalité, il y aurait peut-être moins une branche militaire du Hezbollah, qu'un Hezbollah qui serait le prolongement politique d'une Résistance islamique au Liban (RIL), militaire ${ }^{12}$.

Si distinguer les politiques des militaires apparaît formel, les conséquences sont bien réelles. Les diplomates canadiens et américains au Liban ne peuvent rencontrer des membres du Hezbollah : ni ministres, ni députés. Il n'y a strictement aucun canal de communication. Les pays européens, en faisant une différenciation entre le politique et le militaire qui relève sans doute d'une certaine casuistique, préservent une carte utile : ils peuvent dialoguer directement avec le Hezbollah. Ce dialogue passe par le bloc parlementaire de l'organisation chiite : en juillet 2016, le ministre des Affaires étrangères français, Jean-Marc Ayrault, lors d'une courte visite au Liban, s'est entretenu avec Ali Fayad, un député du Hezbollah également membre du Bureau politique du parti. Déjà, en juillet 2007, Bernard Kouchner avait invité l'ensemble des paris politiques libanais - dont le Hezbollah- à une conférence de dialogue à Saint Cloud. La direction du parti est depuis longtemps rompue aux exercices diplomatiques: elle a ses canaux de discussion avec les Suisses, les Allemands, et le Vatican, le Saint-Siège ayant officiellement accueilli des délégations du Hezbollah par le passé.

Classer le Hezbollah sur la liste des organisations terroristes sert à durcir le ton, à l'heure du conflit syrien. Mais les pays membres de l'Union européenne, en distinguant militaires et politiques, adoptent un principe de réalité. Le Hezbollah a ses armes : il a aussi ses députés, ses ministres, ses mairies de coalition avec le mouvement chiite Amal de Nabi Berri - le président du Parlement. C'est un parti fortement institutionnalisé. Sans doute certains pays membres de l'UE sont conscients que la logique éradicatrice serait contre-productive, et engagerait le Liban dans une voie difficile, le système libanais étant fondé sur l'idée de compromis communautaire, qui a finalement fonctionné : le Hezbollah a marqué des points en soutenant l'arrivée à la présidence de la République de Michel Aoun en décembre 2016, tout en concédant à ses adversaires du Courant du futur, sunnite, le droit à prendre les rennes d'un gouvernement dont les prérogatives ont été fortement renforcées sur celle de la présidence depuis l'avènement de la seconde République libanaise, en 1989. Les pays membres de l'Union européenne cachent rarement leur inimitié pour le Parti de Dieu. Ils aimeraient sans doute le voir plus affaibli à l'avenir : mais ils doivent composer avec lui.

\section{Le Hezbollah : un principe de légitimité}

Si la parole était à la défense, alors les avocats du Hezbollah ne réfuteraient pas seulement l'accusation de terrorisme. Ils ne se limiteraient pas non plus à retourner le stigmate, et à

12Aurélie Daher, Le Hezbollah : mobilisation et pouvoirs, Presses universitaires de France, Paris, 2014, pp 6795. 
affubler leur adversaire principal du même terme - en l'occurrence Israël. Ils s'appuieraient finalement sur un principe de légitimité. En somme, du point de vue du Hezbollah, ses armes sont tout à fait légales.

\section{Une logique de protection}

Le Hezbollah n'est pas exactement un Etat dans l'Etat. Il a au contraire un rapport fort à l'Etat, et sa narration est la suivante : le Hezbollah protège les frontières de l'Etat, et donc l'Etat se doit de protéger le Hezbollah. Député du parti au Parlement libanais, Hassan Fadlallah rappelle que les différents gouvernements investis depuis 1990 ont tous intégré à leur déclaration d'investiture un «droit à la résistance » : même si le Hezbollah n'est pas nommé directement dans les différentes feuilles de route gouvernementales, il a toujours bénéficié d'une couverture étatique. Hassan Fadlallah distingue plusieurs étapes depuis les années $1990^{13}$ :

De 1990 à 1992, les gouvernements de Selim al-Hoss, Rachid al-Sulh et Omar Karameh reconnaissent alors «le droit du Liban, de son gouvernement et de son peuple à combattre l'occupation (israélienne) par tous les moyens (bi-kull al-Wasa'il), en se basant sur la Charte des Nations unies ». De la tenue des élections législatives libanaises de 1992, sous la tutelle syrienne, jusqu'à la libération du sud-Liban en mai 2000, les gouvernements de Selim al-Hoss et de Rafiq Hariri mentionnent, dans leurs déclarations gouvernementales successives, la résistance à l'occupation comme «la priorité des priorités ». A la suite du retrait israélien de 2000, le gouvernement de Rafiq Hariri rappelle que «l'un des plus important but ( $d u$ gouvernement) est la préservation de la réalisation de la résistance (Hifadh 'ala Injaz alMuqawama) » : le Hezbollah peut garder ses armes, puisqu'Israël occupe toujours, à la suite de son retrait, les Fermes de Chebaa, les collines de Kfar Chouba et les hameaux de Ghajar au sud-Liban. Qui plus est, il y a encore à l'époque des prisonniers libanais en Israël.

Cependant, à partir de mai 2000, le consensus libanais sur les armes du Hezbollah se brise peu à peu. Néanmoins, à la suite du retrait syrien de 2005, le Parti de Dieu continue de bénéficier d'une couverture gouvernementale. Sous la présidence de Michel Sleiman (2007), un nouveau compromis s'établit entre le Hezbollah et l'Etat: c'est le fameux triptyque associant «le peuple, l'armée, et la résistance». En 2013, le gouvernement de Tammam Salam mentionne «un droit des citoyens libanais à la résistance contre l'occupation israélienne et à répondre aux agressions ». En décembre 2016, c'est la présidence de la République qui appuie les positions du Hezbollah: dans son discours d'investiture présidentielle, Michel Aoun dit ne «vouloir épargner aucun effort ni aucune résistance afin de libérer ce qui reste comme territoire libanais ${ }^{14} \gg$.

\section{Un contrat entre le Hezbollah et l'Etat libanais}

Les opposants au Hezbollah n'ont eu de cesse de dénoncer, depuis mai 2000, les armes du Hezbollah. Elles seraient illégitimes pour plusieurs raisons : les derniers prisonniers libérés par Israël l'ont été en juillet 2008. Les Fermes de Shebaa, encore occupées par Israël, ne seraient pas libanaises, mais syriennes. Le Hezbollah aurait retourné ses armes contre des Libanais, lors de «la prise de Beyrouth» de mai 2008. Enfin, son intervention en Syrie depuis 2012 l'aurait détourné de son combat contre Israël.

De son côté, le Hezbollah se prévaut d'un strict principe de légitimité : il ne serait pas un parti terroriste, puisqu'il bénéficierait depuis 1990 d'une couverture gouvernementale et

\footnotetext{
${ }^{13}$ Hassan Fadlallah, Hezbollah wa-l-Dawla fi-Lubnan. Al-Ru'waya wa-l-Massar (Le Hezbollah et l'Etat au Liban. Visions et cheminement), Sharika al-Matbu'at li-l-Tawzi' wa-l-Nashr, Beyrouth, 2015, pp 105-108. Les citations tirées des déclarations gouvernementales sont issues de cet ouvrage.

${ }^{14}$ L'Orient le Jour, « Le gouvernement adopte la déclaration ministérielle », 24 décembre 2016.
} 
présidentielle quasi-systématique - il s'inscrirait donc dans une certaine légalité libanaise. Il aurait toujours collaboré avec l'armée. Cette dernière, trop faible, dépourvue d'aviation et de marine dignes de ce nom, n'aurait pas les moyens d'établir un équilibre stratégique dissuasif face à Israël. La logique du Hezbollah, c'est donc celle du contrat : l'Etat protège les armes de la résistance, et en retour la résistance protège la souveraineté de l'Etat. C'est un contrat parfois précaire, soumis à une triple critique : de la Coalition du 14 mars, des principales puissances occidentales - qui se prévalent d'un autre ordre du droit, à savoir le droit international et la résolutions du Conseil de sécurité 1559, et, depuis 2011, de l'opposition syrienne à Bashar al-Assad, qui dénonce la présence du Hezbollah en Syrie comme une forme d'occupation étrangère.

Or, c'est aujourd'hui précisément cette présence en Syrie qui permet au Hezbollah d'intégrer la rhétorique de l'anti-terrorisme à son arsenal conceptuel. Depuis 2012, le Hezbollah a utilisé trois arguments majeurs pour justifier son entrée en guerre aux côtés de Bashar al-Assad. La rhétorique confessionnelle et religieuse n'est pas la principale, mais elle existe : défendre les lieux saints chiites en Syrie, comme le sanctuaire de Saida Zaynab à Damas, mais aussi des villages chiites libanais situés sur les frontières syro-libanaises. L'argument géopolitique est par contre central : il existe "un axe de la résistance » (Mihwar al-Muqawama) passant par Damas, Beyrouth, Gaza et Téhéran : si Bashar al-Assad s'est tenu aux-côtés du Hezbollah lors de la guerre de juillet et août 2006 contre Israël, alors le Hezbollah doit se tenir maintenant aux-côtés de Bashar al-Assad. Le fait que l'armée israélienne ait plusieurs fois attaquée des positions militaires du Hezbollah sur le Plateau du Golan syrien et ait assassiné certains de ses dirigeants à Damas, permet enfin au Hezbollah de raccorder deux narrations : la lutte contre Israël, et le combat contre les forces de l'opposition syrienne.

Enfin, la nature radicale et «takfiristes » de groupes comme le Fatah al-Sham - anciennement lié à Al-Qaeda- et l'Etat islamique autoriserait le Hezbollah à intervenir en Syrie, en vertu d'une guerre préventive, que le Parti de Dieu décrit également comme "une guerre défensive » (Harb difa 'iya) ${ }^{15}$ : le Hezbollah défendrait l'intégrité des frontières orientales du Liban. La vaste offensive lancée par le Hezbollah, le 21 juillet 2017, dans la région de Ersal, sur la frontière syro-libanaise, contre les troupes du Fatah al-Sham et de l'Etat islamique, a valeur d'exemple: appuyé par l'armée syrienne, mais coordonnant ses mouvements avec l'armée libanaise, le Hezbollah se présente au final comme le premier rempart à la poussé djihadiste, et comme un élément moteur d'une nouvelle équation anti-terroriste régionale ${ }^{16}$.

\section{Un cas d'école}

Pour les uns, le Hezbollah déstabiliserait la Syrie, menacerait Israël, et ne défendrait que les intérêts iraniens. Au mieux, il faudrait l'éradiquer, comme le souhaiteraient Washington, Tel Aviv et Ryad; au pire, il faudrait en limiter l'expansion, en distinguant, en son sein, les militaires et les politiques, comme le fait l'Union européenne. Pour d'autres, le Hezbollah est un parti nationaliste : il sécurise des frontières. Il a libéré le sud du Liban de l'occupation israélienne, établi un équilibre de la terreur face aux Israéliens depuis 2006, endigué la prolifération des groupes djihadistes depuis 2012. Les adversaires du Hezbollah le décrivent

\footnotetext{
${ }^{15}$ Hassan Fadlallah, Hezbollah wa-l-Dawla fi-Lubnan. Al-Ru'waya wa-l-Massar (Le Hezbollah et l'Etat au Liban. Visions et cheminement), opus cité, p 224.

${ }^{16}$ Chiara Calabrese, «La cause c'est nous : militants du Hezbollah face à la guerre en Syrie depuis 2011 », in Partis et partisans dans le monde arabe post-2011, sous la direction de Robin Beaumont et Xavier Guignard, revue Confluences Méditerranée, numéro 98, L’Harmattan/ Iremmo, Paris, automne 2018.
} 
comme un mouvement terroriste empêchant le Liban de recouvrer une véritable souveraineté nationale : ses armes interdiraient à l'Etat libanais tout «monopole de la violence légitime». Les partisans du Hezbollah disent exactement le contraire : il est un élément moteur d'une équation anti-terroriste l'associant de manière étroite aux organes sécuritaires libanais services de renseignements de l'armée, Forces de sécurité intérieure (FSI), Sûreté générale.

Les prédictions de 2011 se sont effondrées : le Hezbollah n'a pas été affaibli par son soutien à un régime syrien résilient. Les Israéliens reconnaissent d'ailleurs qu'il s'est renforcé ${ }^{17}$. Sur le plan intérieur, il est soumis à des pressions moindres que lors de la seconde moitié des années 2000 : la présidence de la République lui est pour le moment favorable, et la coalition du 14 mars participe à un gouvernement d'union nationale à ses côtés. Les principaux pays occidentaux, quant à eux, sont gênés aux entournures. Ils peuvent s'opposer au Hezbollah, au moins verbalement, sinon financièrement, mais ils ne peuvent plus le confronter : comment affaiblir un mouvement dit terroriste, mais bien implanté dans l'Etat libanais, et qui mène une bataille anti-terroriste majeure contre l'Etat islamique ? Déstabiliser le Hezbollah reviendrait alors, dans la conjoncture actuelle, à déstabiliser le Liban. Le problème est donc insoluble.

Car le Hezbollah n'est pas un Etat dans l'Etat, contrairement à un sentiment répandu, mais une organisation tout à la fois à-côté et aux-côtés de l'Etat. C'est moins qu'une armée régulière, et plus qu'un mouvement de guérilla. C'est une formation nationale, mais il a une fonction régionale. C'est un mouvement islamiste, mais qui ne prétend pas bâtir un Etat islamique au Liban. Il est chiite, mais il finance, arme et entraîne des Palestiniens sunnites. Pour le Conseil de sécurité de l'ONU, il est sorti du droit international; mais au Liban, il se prévaut d'un principe de légalité. Incontestablement résilient, le Hezbollah est devenu un cas d'école.

${ }^{17}$ Anat Kurtz and Schlomo Brom (Editors), Strategic Survey for Israel 2016-2017, The Institute for National Security Studies, Tel Aviv University, 2017, http://www.inss.org.il/wp-content/uploads/2017/01/full.pdf 